\title{
Straightforward Probing of Polarization and Spin-Resolved Transmission through Potential Barriers in Silicene
}

\author{
Diana Meneses-Gustin and Marcos R. S. Tavares \\ Centro de Ciências Naturais e Humanas, Universidade Federal do ABC, 09210-170 Santo André, SP, Brazil \\ Correspondence should be addressed to Marcos R. S. Tavares; mdas.tavares@gmail.com
}

Received 16 July 2017; Accepted 9 December 2017; Published 18 March 2018

Academic Editor: Joseph S. Poon

Copyright (c) 2018 Diana Meneses-Gustin and Marcos R. S. Tavares. This is an open access article distributed under the Creative Commons Attribution License, which permits unrestricted use, distribution, and reproduction in any medium, provided the original work is properly cited.

\begin{abstract}
The low-energy physics of silicene is described by Dirac electrons with a strong spin-orbit interaction and its band structure can be controlled by an external electric field $E_{z}$. We evaluate the electronic transmission through single and double barriers in silicene as a function of the angle of incidence $\theta$, the electron energy $E$, and the strength of the field $E_{z}$. We find a spin-resolved transmission, reflected in the polarization $P$, and a conductance qualitatively different in some ranges of $E$ than that in graphene. $P$ exhibits quasiperiodic resonances versus $\theta$ in double-barrier structures.
\end{abstract}

\section{Introduction}

The specific two-dimensional (2D) material, a monolayer honeycomb structure of silicon, called silicene, has been recently synthesized $[1,2]$ and attracted, as usual, considerable attention [3]. The reason for that is that silicene has Dirac cones mostly similar to those in graphene, which is the genesis of 2D materials achieved in laboratory. In addition, and contrary to graphene in which the spin-orbit interaction (SOI) is very weak, silicene has a strong SOI that leads to a gap of $1.55 \mathrm{meV}$. This gap can be further controlled by an external electric field $E_{z}$ and is facilitated by the buckled structure of silicene resulting from the large ionic radius of silicon. This and its compatibility with silicon-based electronic technology led to important and seminal studies such as the spin-Hall effect [4], the anomalous Hall effect [5, 6], and the capacitance of an electrically tunable silicene device [7].

Since the SOI can lead to spin-resolved transport, pertinent to quantum computing and other important issues, it is worth studying SOI further in silicene and eventually contrast these results with graphene's $[8,9]$. We then simply and carefully undertake such a study by evaluating both the electronic transmission and polarization through single and double potential barriers in silicene as a function of the electron's angle of incidence $\theta$, its energy $E$, and the strength of the external field $E_{z}$. It was suggested that $E_{z}$, solely, might not be enough to lift spin resolution in silicene, and an external magnetic field should be present in order to locally break the time-reversal symmetry [10]. But to avoid including further physical parameters such as the magnetic field, which surely brings extra physical nuances to the calculation, we choose to look at one valley only and probe the eventual spin polarization which certainly appears in the presence of magnetic field. In this way, we present a simpler (but scholarly sound) way to probe the spin resolution, which is, by virtue of the SOI, an inherent feature of these novel materials. Furthermore, we calculate the spin polarization for the double-barrier case. This polarization presents structures showing many resonance features which can be also analyzed by virtue of the evaluated polarization measured in laboratory.

In fact, broader studies [11-14] on 2D Si-based devices have shown important features concerning their timely usage, contribution to the future of both theoretical and experimental investigations, and their correspondent application on technology industry. In these previous works, some interesting issues on magnetic and quantum topological states have been reported. The present work also serves as a primary comparison to such results. 
The manuscript is organized as follows. Section 2 is devoted to the theoretical formulation. Sections 3 and 4 explore, respectively, the pertinent cases of single and doublebarrier structures. Our conclusions are given by comparing Sections 3 and 4 .

\section{Formalism}

The one-electron Hamiltonian for carriers in a single $K$ valley in silicene is given by the following [4]:

$$
\begin{aligned}
H_{K} & =\left(\begin{array}{ll}
H_{++} & H_{+-} \\
H_{-+} & H_{--}
\end{array}\right) \\
& =\left(\begin{array}{cccc}
s_{-} & \hbar v_{F} k_{-} & i a \lambda_{R 2} k_{-} & 0 \\
\hbar v_{F} k_{+} & -s_{-} & -i \lambda_{R 1} & -i a \lambda_{R 2} k_{+} \\
-i a \lambda_{R 2} k_{+} & i \lambda_{R 1} & -s_{+} & \hbar v_{F} k_{-} \\
0 & i a \lambda_{R 2} k_{+} & \hbar v_{F} k_{+} & s_{+}
\end{array}\right),
\end{aligned}
$$

where $v_{F}$ is the Fermi velocity, $a=0.386 \mathrm{~nm}$ the lattice constant, and $k_{ \pm}=k_{x} \pm i k_{y}$. Also, $s_{ \pm}=\lambda_{\text {SOI }} \pm \ell E_{z}$ where $\lambda_{\text {SOI }} \equiv \lambda \simeq 3.9 \mathrm{meV}$ is the strength of the SOI in the lattice and $2 \ell$ is the distance between the two sublattices $A$ and $B$. It has been noticed that the secondary (Rashba) spin coupling parameters $\lambda_{R 1}=10 \mu \mathrm{eV}$ and $\lambda_{R 2}=0.7 \mathrm{meV}$ are too small in comparison to the primary one $\lambda_{\text {SOI }}$. Here $R 1$ and $R 2$ stand for two types of the Rashba spin coupling often appearing in the silicene Hamiltonian. These parameters are negligible compared to the spin-orbit one, $\lambda_{\mathrm{SOI}}$. Therefore, we hereafter deliberately assume $H_{-+}=H_{+-} \simeq 0$ without any significantly loss of science.

The resulting matrix is then block-diagonal with its upper (lower) block pertaining to spin-up (spin-down) electrons. The latter is given by

$$
H_{--}=\left(\begin{array}{cc}
-\lambda-\ell E_{z} & \hbar v_{F} k_{-} \\
\hbar v_{F} k_{+} & \lambda+\ell E_{z}
\end{array}\right)=\left(\begin{array}{cc}
-s_{+} & \hbar v_{F} k_{-} \\
\hbar v_{F} k_{+} & s_{+}
\end{array}\right),
$$

with the Hamiltonian for spin-up electrons $H_{++}$being given by (2) with $s_{+}$replaced with $-s_{-}$. The eigenvectors of (2) are straightforwardly written in the forms $\Psi=\left(\varphi_{A}^{\downarrow}, \varphi_{B}^{\downarrow}\right)^{T}$ for spindown, and $\Psi=\left(\varphi_{A}^{\uparrow}, \varphi_{B}^{\uparrow}\right)^{T}$ for spin-up electrons with $A$ and $B$ labelling the two sublattices and $T$ denoting the transpose. With $\mu= \pm$, the corresponding eigenvalues are

$$
E_{k \mu}=\mu\left[\hbar^{2} v_{F}^{2} k^{2}+s_{ \pm}^{2}\right]^{1 / 2} \text {. }
$$

We then leave the details of eigenvectors of (2) for the next sections where we study the single and double barriers scholar problems.

\section{Single Barriers}

In this section, we consider a single barrier of a constant height $U$ and of width $W$, along the $x$-axis and infinitely long along the $y$-axis. This barrier is schematically shown in Figure 1. Such a constant potential $U(x)$ is subjected then

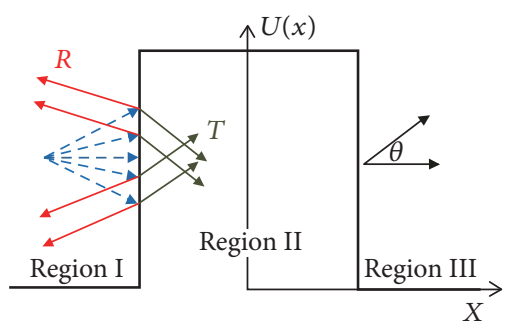

FIGURE 1: (Color online) Schematically potential profile showing regions I, II, and III and their relationship between the incident angle $\theta$ and the eventual transmission $T$ and reflection $R=1-T$ through the barrier [15].

to the free particle Hamiltonian (2). Along the $y$-direction, the eigenvectors are simple plane waves $e^{i k_{y} y}$, provided the electrons are free from any force there. The same happens for the $x$-direction to the left (region I) and to the right (region II) of the barrier. So, for region I, the eigenvectors are written in the form of plane wave:

$$
\varphi_{A}^{\mathrm{I}}=e^{i \eta x}+r e^{-i \eta x}
$$

where $r$ is the reflection amplitude. For region III, it is in the following form:

$$
\varphi_{A}^{\mathrm{III}}=t e^{i \eta x},
$$

with $t$ being the transmission amplitude.

In the barrier (region II), the eigenvector is written as

$$
\varphi_{A}^{\mathrm{II}}=a e^{i \delta x}+b e^{-i \delta x} .
$$

Here, the parameters $\delta^{2}=\left[(U-E)^{2}-s_{ \pm}^{2}\right] / \hbar^{2} v_{F}^{2}-k_{y}^{2}$, and $\eta^{2}=\left(E^{2}-s_{ \pm}^{2}\right) / \hbar^{2} v_{F}^{2}-k_{y}^{2}$, with $v_{F}$ being the Fermi velocity. We mention, concerning the component $\varphi_{B}$, that, through the diagonalization of (2), one can easily find

$$
\varphi_{B}=i \hbar v_{F} \frac{\left(\partial \varphi_{A} / \partial x-k_{y} \varphi_{A}\right)}{\left(s_{ \pm}+U-E\right)}
$$

from which the component $\varphi_{B}$ can be written for the three barrier regions.

The electronic transmission $T$ is obtained after matching $\varphi_{A}$ and $\varphi_{B}$ at the interfaces between regions I and II and regions II and III. Within such a procedure, the transmission $T=t t^{*}$ takes that standard form obtained whenever the Dirac-like Hamiltonian is used instead of Shröndiger's in calculating $T$. After a straightforward (but quite cumbersome) calculation, the matching conditions lead us to the transmission coefficient for spin-up (+) and spin-down (-) electrons written as

$$
T_{ \pm}=\frac{1}{\left[1+F \sin ^{2}(W \delta)\right]}
$$

with $F=b_{-} b_{+} /\left[2 \eta \delta\left(s_{ \pm}-E\right)\left(s_{ \pm}+U-E\right)\right]^{2}$ and $b_{ \pm}=\left[\eta\left(s_{ \pm}+\right.\right.$ $\left.U-E) \pm \delta\left(s_{ \pm}-E\right)\right]^{2}+v_{F}^{2} k_{y}^{2}$. As in the graphene case [16], we 


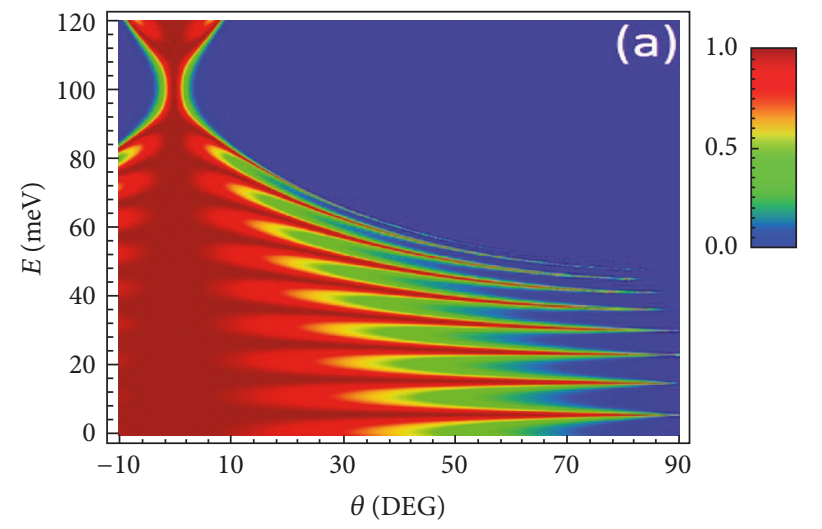

(a)

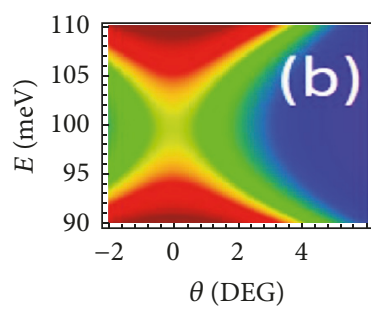

(b)

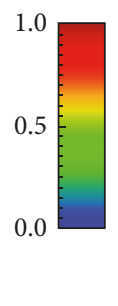

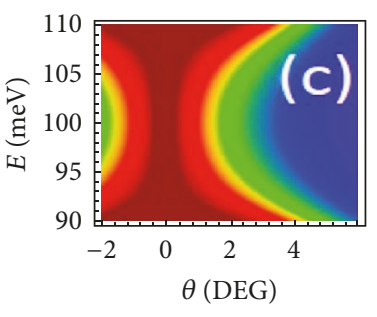

(c)
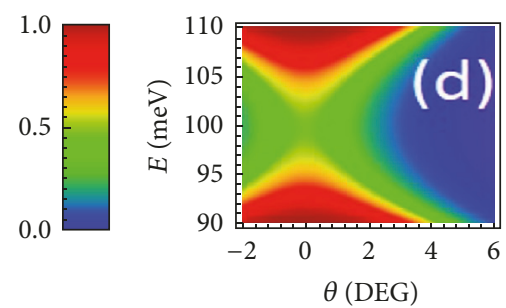

(d)

Figure 2: (Color online) (a) $(E, \theta)$ contour plot of the transmission through a single barrier for spin-up electrons. The width and height of the barrier are $W=110 \mathrm{~nm}$ and $U=100 \mathrm{meV}$, respectively, the field $E_{z}=170 \mathrm{meV} / \mathrm{nm}$, and $v_{F}=5.5 \times 10^{5} \mathrm{~m} / \mathrm{s}$. Panels (b), (c), and (d) show the region $(E, \theta)$ delimited by $-2 \leq \theta \leq 6$ and $90 \leq E \leq 110 \mathrm{meV}$ and correspond, respectively, to $E_{z}=50,170$, and $300 \mathrm{meV} / \mathrm{nm}$.

see $T_{ \pm}$with clear dependence on $k_{y}$, which is absent $\left(k_{y}=0\right)$ in usual semiconductor barriers. But in contrast to it, now $T$ depends on $s_{ \pm}$as well, and, for $s_{ \pm}=0,(8)$ gives the graphene result, naturally, [16] from which one analytically obtains $T=$ 1 for normal incidence $\left(k_{y}=0\right)$. So, the electrons are totally transmitted when they are injected in a normal direction with respect to the barrier.

In Figure 2(a) we show a $(E, \theta)$ contour plot of the transmission $T_{\uparrow}$ for spin-up electrons for a single barrier. The width and height of the barrier are $W=110 \mathrm{~nm}$ and $U=$ $100 \mathrm{meV}$, respectively. Intentionally, we set $E_{z}=170 \mathrm{meV} / \mathrm{nm}$ because this value of $E_{z}$, together with $\ell=0.23 \AA$ and $\lambda_{\text {SOI }}=$ $3.9 \mathrm{meV}$, gives $s_{-}=0$ and makes the diagonal terms in (2) vanish. In this case we should obtain graphene's results [16] for $v_{F}=10^{6} \mathrm{~m} / \mathrm{s}$. This is indeed the case.

The plotted results though differ very slightly because we used $v_{F}=5.5 \times 10^{5} \mathrm{~m} / \mathrm{s}$ appropriate for silicene. The question then arises what influence changing $E_{z}$ has on the results. To partially address that and in view of Figure 3 we focus on the region of the $(E, \theta)$ plane approximately delimited by $-2 \leq$ $\theta \leq 6$ and $90 \leq E \leq 110 \mathrm{meV}$, in panels (b), (c), and (d) that correspond, respectively, to $E_{z}=50,170$, and $300 \mathrm{meV} / \mathrm{nm}$. As can be seen, for values of $E_{z}$ away from the "graphene" value $E_{z}=170 \mathrm{meV} / \mathrm{nm}$ the perfect transmission weakens considerably.

We now consider spin-down electrons and show in Figure $3(\mathrm{a})$ the corresponding $(E, \theta)$ contour plot of the transmission.
The results are similar to those in Figure 2(a) for most angles of incidence $\theta$ except for normal or near-normal incidence, that is, for $\theta \approx 0$. In this case, we have a gap in the transmission approximately for $90 \leq E \leq 110 \mathrm{meV}$ (Eq. (3) for $k y=0$ gives $T=0$ for $E=V \pm s+$ and $E=s+$ ). Together with Figure 2 this means that we have a spin-resolved transmission.

Again, to assess the influence of the field $E_{z}$, we show in panels (b), (c), and (d) a small region containing the gap for the same values of $E_{z}$ as in Figure 2. As can be seen, the main effect of increasing $E_{z}$ from its "graphene" value $E_{z}=170 \mathrm{meV} / \mathrm{nm}$ is to increase the gap, while for $E_{z}$ less than this value, the gap tends to close. We notice in passing that upon reversing the field $E_{z}$ the factor $s_{+}$in (2) becomes $s_{-}$and the up spins will be blocked, as in Figure 3, whereas the down spins $\left(s_{-} \rightarrow s_{+}\right)$will be transmitted as in Figure 2 .

With the results of Figures 2(a) and 3(a) we show in Figure $4(\mathrm{a})$ a $(E, \theta)$ contour plot of the polarization $P$ defined by $P=\left(T_{\uparrow}-T_{\downarrow}\right) /\left(T_{\uparrow}+T_{\downarrow}\right)$. $P$ vanishes in most of the $(E, \theta)$ plane. Notice, however, the regions where it does not and especially its $100 \%$ value for near-normal incidence and $90 \leq$ $E \leq 110 \mathrm{meV}$, which corresponds to that of the transmission gap in Figure 3(a). This is drastically different from the case of graphene in which $P$ vanishes everywhere due to the smallness of $\lambda_{\text {SOI }}$ and the vanishing of the distance $\ell$ between the two sublattices. This difference remains as sharp when we reduce the height $U$. The plot in Figure 4(b) is drawn for the same values of $W$ and $E_{z}$ as in (a) but for $U=50 \mathrm{meV}$. As 

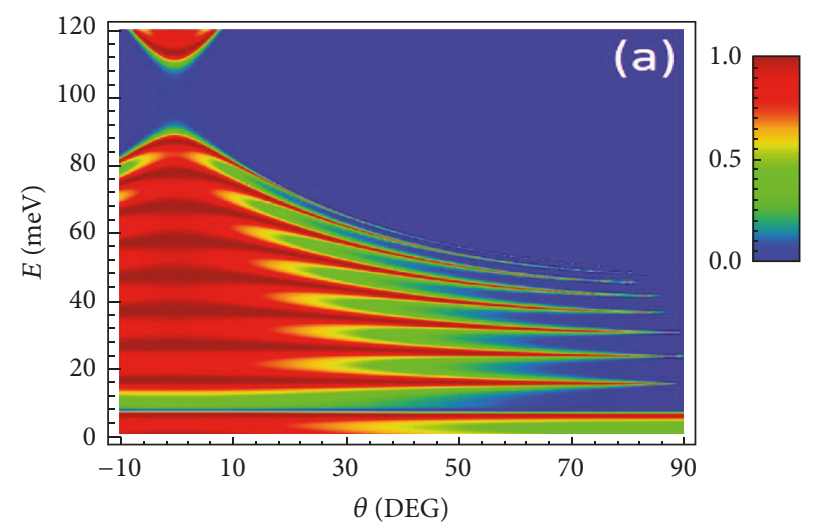

(a)

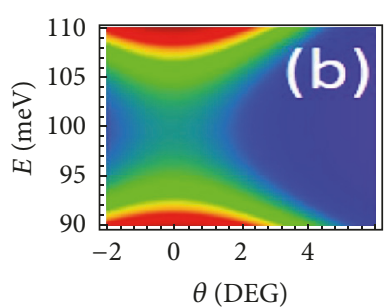

(b)
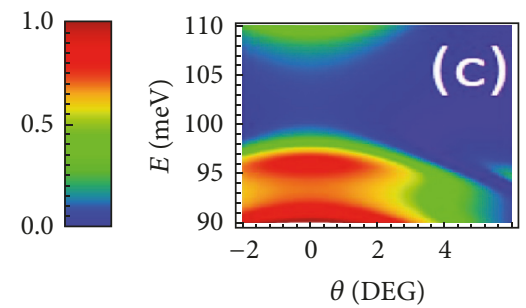

(c)
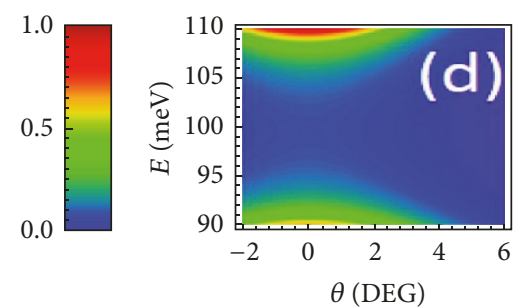

(d)

FIGURE 3: As in Figure 2 for spin-down electrons.

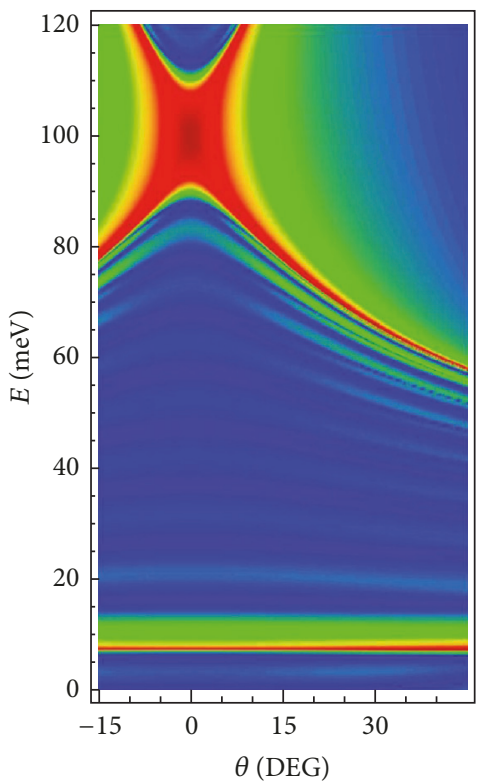

(a)
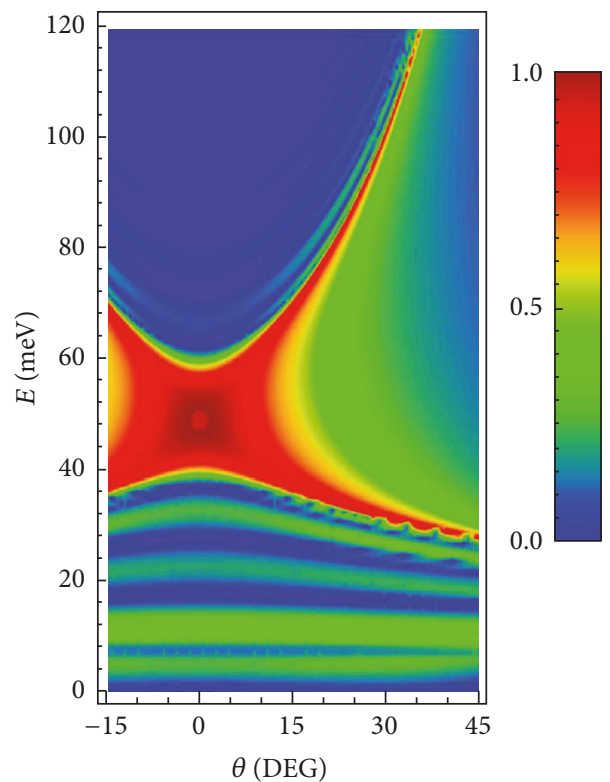

(b)

FIgURE 4: (Color online) (a) Contour plot of the polarization for a single barrier using the results of Figures 2(a) and 3(a). (b) As in (a) with $U=50 \mathrm{meV}$.

shown, the most important change is that the "red" window in (a), centred at $\theta=0, U=100 \mathrm{meV}$, is now centred at $\theta=0$, $U=50 \mathrm{meV}$, and nearly twice as wide.

Again, in there $T_{\uparrow}$, controlled by the "graphene" value of $E_{z}$, is almost 1 and $T_{\downarrow} \approx 0$. For the same $U$ and $E_{z}$ but different $W$, however, the changes are more substantial; details will be given elsewhere.

\section{Double Barriers}

In this case, the analytic result for the transmission is very unwieldy and will not be given. We present numerical results for spin-up electrons in Figure 5 with the parameters specified in its caption. Again for $E_{z}=170 \mathrm{meV} / \mathrm{nm}$, we have the results for graphene for a $\left(k_{y}, k_{x}\right)$ contour plot, since the 


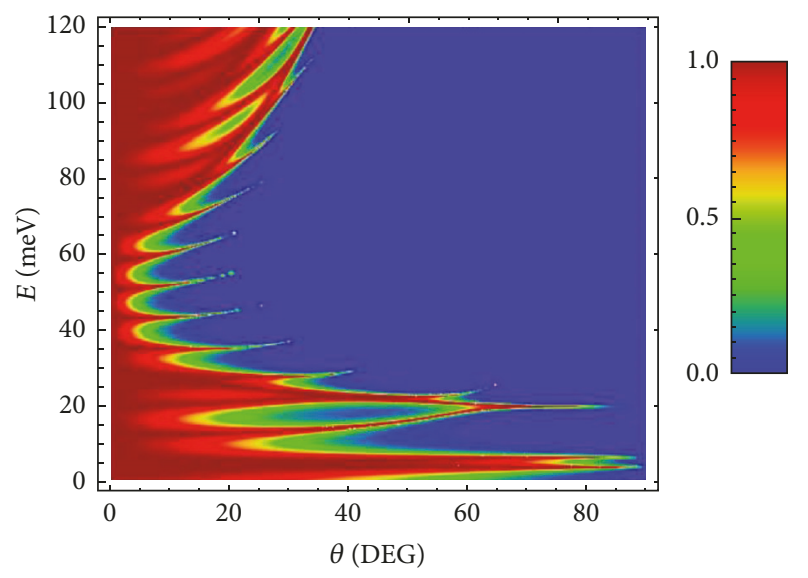

Figure 5: (Color online) (a) $(E, \theta)$ contour plot of the transmission through a double barrier for spin-up electrons. The width and height of the barriers are $W=50 \mathrm{~nm}$ and $U=50 \mathrm{meV}$, respectively, the interbarrier separation $d=100 \mathrm{~nm}$, and the field $E_{z}=170 \mathrm{meV} / \mathrm{nm}$.

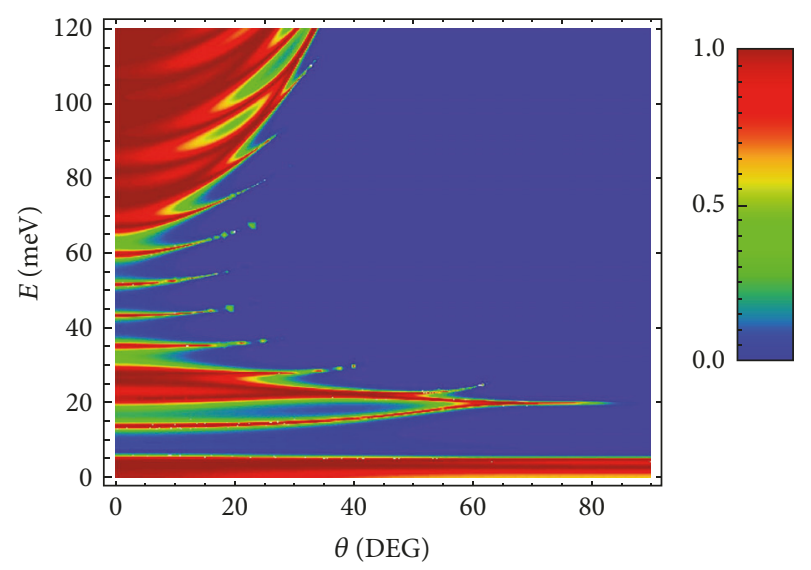

FIgURE 6: As in Figure 5 for spin-down electrons.

diagonal terms in (2) vanish. As in that case we see many more resonances than in single barriers. Upon contrasting these results, though, with those of Figure 6 for spin-down electrons, we see again qualitative differences especially for near-normal incidence with several gaps in the transmission. That is, we have a spin-resolved transmission that is more complex than that for single barriers. Again, this structure of the transmission is reflected in the $(E, \theta)$ contour plot of the polarization $P$ that we show in Figure 7.

Notice the quasiperiodic character of $P$ versus the angle $\theta$ for energies, say, larger than $80 \mathrm{meV}$. This contour plot contrasts rather sharply with that of Figure 4 for single barriers as we now see several windows of perfect polarization especially for near-normal incidence.

The results presented so far raise the question of their sensitivity to the parameters. We emphasize that all contour plots for double barriers are sensitive to their height $U$ and the distance $d$ between them. This is expected since more resonances can be created by changing $U$ and $d$. The results for single barriers show a similar but weaker sensitivity (cf.

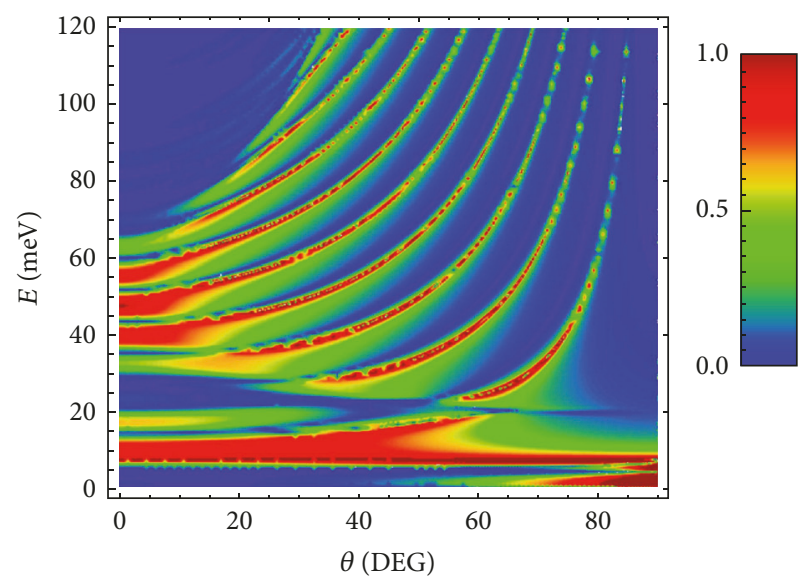

Figure 7: (Color online) Polarization for a double barrier.

Figures 3(a) and 3(b)). A detailed account of these sensitivities will be reported elsewhere.

Another concern about the results is that experimentally one usually measures the current, which is proportional to a weighted integral of the transmission. One then may wonder to what extent the spin-resolved features survive after such an averaging. We show in Figure 8 the conductance $G$ using the standard formula:

$$
G=\left(\frac{e^{2}}{\hbar}\right) \int T(E, \theta) E \cos \theta d \theta .
$$

The red, dashed curve is for spin-up $\left(G_{\uparrow}, s_{-}=0\right)$ electrons and the blue, solid one for spin-down $\left(G_{\downarrow}, s_{+} \neq 0\right)$ electrons.

As shown in Figure 8(a), near the 2nd minimum of the two curves, we have a gap in $G_{\downarrow}$, as well as a dip for $E=$ $10 \mathrm{meV}$, in agreement with Figure 3. In general, relative to graphene, the overall silicene results for $G$ are similar but one sees important qualitative differences as well. This is more pronounced for double barriers for which $G_{\downarrow}$, pertinent to Figure 6, has four very deep minima or gaps as Figure 8(b) demonstrates. All gaps in Figure 8 could get wider by increasing the value of $E_{z}$ (cf. Figures 3(a) and 3(d)).

As one can notice, this work has not considered substrate effects, which is a very interesting issue to tackle theoretically $[13,14]$. It is well know that the subtracts can even destroy the $2 \mathrm{D}$ feature of these materials. Indeed, this happened in the earlier graphene samples, where the Dirac cones vanish if the material was hosted by certain substrates. In some cases a SOI like parameter could mimic the coupling between the Dirac electrons and the substrate, since it recovers the massive Schrödinger behavior for the Dirac electrons in graphene $[8,9]$. As a matter of fact, the concern on how to select the suitable substrate to keep the $2 \mathrm{D}$ features intact is actually an open experimental issue which is certainly beyond the scope of this paper. But, we emphasize that our results are very reliable to easily probe the spin polarization strength by simply considering one $\mathrm{K}$ valley only in the $2 \mathrm{D}$ silicene.

In summary, we evaluated the electronic transmission through single and double barriers in silicene as a function of the angle of incidence $\theta$, the electron energy $E$, and the 


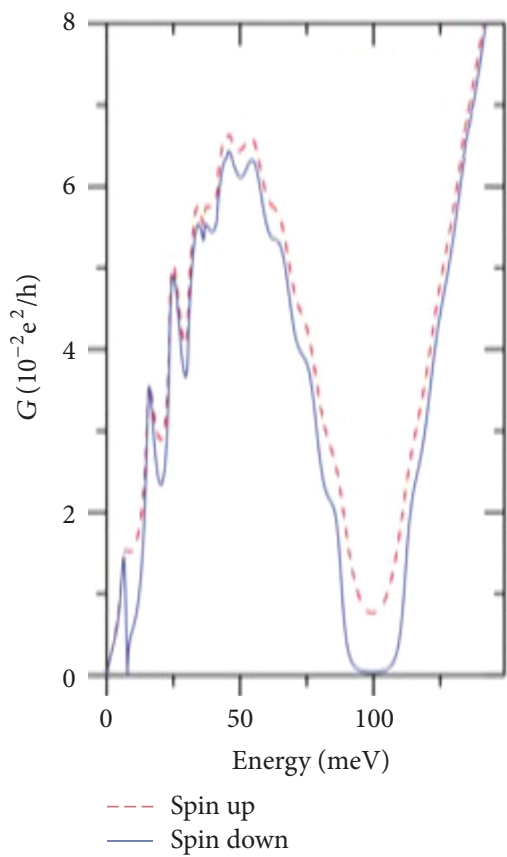

(a)

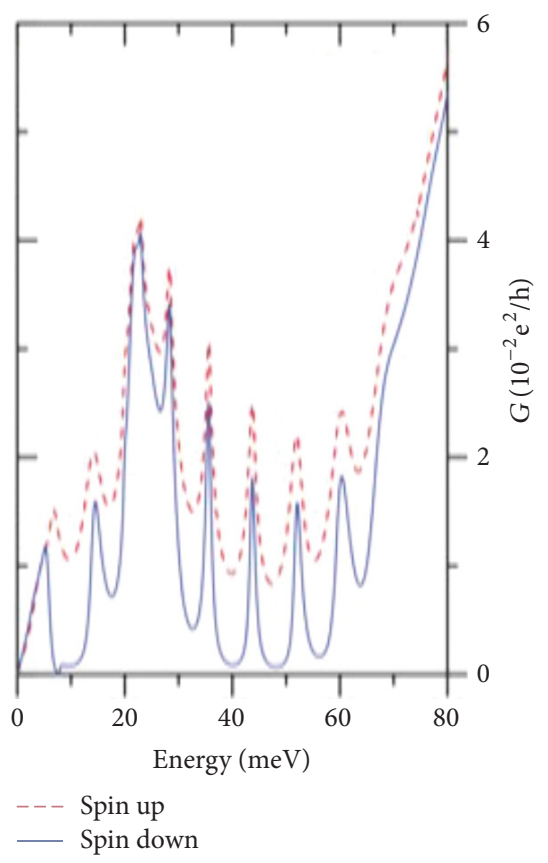

(b)

Figure 8: (Color online) (a) Conductance through a single barrier versus energy $E$. The dashed and solid curves are for spin-up $\left(G_{\uparrow}, s_{-}=0\right)$ and spin-down $\left(G_{\downarrow}, s_{+} \neq 0\right)$ electrons, respectively. (b) Conductance through the double barrier of Figures 5-7. The Fermi velocity (energy) is given in the caption of Figure 2.

strength of the field $E_{z}$ and obtained a spin-resolved transmission, especially for near-normal incidence, and a conductance qualitatively different in some ranges of $E$ than that in graphene. The strength of the resolution depends on the barrier parameters. This is entirely due to the strong SOI in silicene and does not occur in graphene in which the SOI is very weak. The results may be useful in spintronics.

\section{Conflicts of Interest}

The authors declare that they have no conflicts of interest.

\section{Acknowledgments}

This work was supported by Universidade Federal do ABC and CNPq (Brazil), no. 131134/2012-4.

\section{References}

[1] P. Vogt, P. De Padova, C. Quaresima et al., "Silicene: compelling experimental evidence for graphenelike two-dimensional silicon," Physical Review Letters, vol. 108, no. 15, Article ID 155501, 2012.

[2] A. Fleurence, R. Friedlein, T. Ozaki, H. Kawai, Y. Wang, and Y. Yamada-Takamura, "Experimental evidence for epitaxial silicene on diboride thin films," Physical Review Letters, vol. 108, no. 24, Article ID 245501, 2012.

[3] Z. Ni, Q. Liu, K. Tang et al., "Tunable bandgap in silicene and germanene," Nano Letters, vol. 12, no. 1, pp. 113-118, 2012.
[4] C.-C. Liu, W. Feng, and Y. Yao, "Low-energy effective Hamiltonian involving spin-orbit coupling in silicene and twodimensional germanium and tin," Physical Review B, vol. 84, no. 19, Article ID 195430, 2011.

[5] M. Ezawa, "Valley-polarized metals and quantum anomalous hall effect in silicene," Physical Review Letters, vol. 109, no. 5, Article ID 055502, 2012.

[6] M. Ezawa, "A topological insulator and helical zero mode in silicene under an inhomogeneous electric field," New Journal of Physics, vol. 14, Article ID 033003, 11 pages, 2012.

[7] M. Tahir and U. Schwingenschlögl, "Magnetocapacitance of an electrically tunable silicene device," Applied Physics Letters, vol. 101, no. 13, Article ID 132412, 2012.

[8] C. E. Villegas and M. R. Tavares, "Controlling the current flux in magnetic-barrier induced graphene waveguide," Applied Physics Letters, vol. 101, no. 16, p. 163104, 2012.

[9] C. E. P. Villegas, M. R. S. Tavares, G.-Q. Hai, and F. M. Peeters, "Sorting the modes contributing to guidance in strain-induced graphene waveguides," New Journal of Physics, vol. 15, Article ID 023015, 2013.

[10] W.-F. Tsai, C.-Y. Huang, T.-R. Chang, H. Lin, H.-T. Jeng, and A. Bansil, "Gated silicene as a tunable source of nearly $100 \%$ spinpolarized electrons," Nature Communications, vol. 4, article 1500, 2013.

[11] C.-W. Zhang and S.-S. Yan, "First-principles study of ferromagnetism in two-dimensional silicene with hydrogenation," The Journal of Physical Chemistry C, vol. 116, no. 6, pp. 4163-4166, 2012.

[12] J. S. Kim, H. W. Jeong, W. Lee, B. G. Park, B. M. Kim, and K. B. Lee, "A simple and fast fabrication of a both self-cleanable and deep-UV antireflective quartz nanostructured surface," Nanoscale Research Letters, vol. 7, 2012. 
[13] R. Zhang, C. Zhang, W. Ji et al., "Silicon-based chalcogenide: Unexpected quantum spin Hall insulator with sizable band gap," Applied Physics Letters, vol. 109, no. 18, p. 182109, 2016.

[14] R.-W. Zhang, C.-W. Zhang, W.-X. Ji et al., "Silicane as an inert substrate of silicene: A promising candidate for FET," The Journal of Physical Chemistry C, vol. 118, no. 43, pp. 2527825283, 2014.

[15] R. N. Sajjad and A. W. Ghosh, "Silicon-based chalcogenide: Unexpected quantum spin Hall insulator with sizable band gap," Applied Physics Letters, vol. 99, no. 18, Article ID 123101, 2011.

[16] M. Barbier, P. Vasilopoulos, and F. M. Peeters, "Single-layer and bilayer graphene superlattices: Collimation, additional Dirac points and Dirac lines," Philosophical Transactions of the Royal Society A: Mathematical, Physical \& Engineering Sciences, vol. 368, no. 1932, pp. 5499-5524, 2010. 

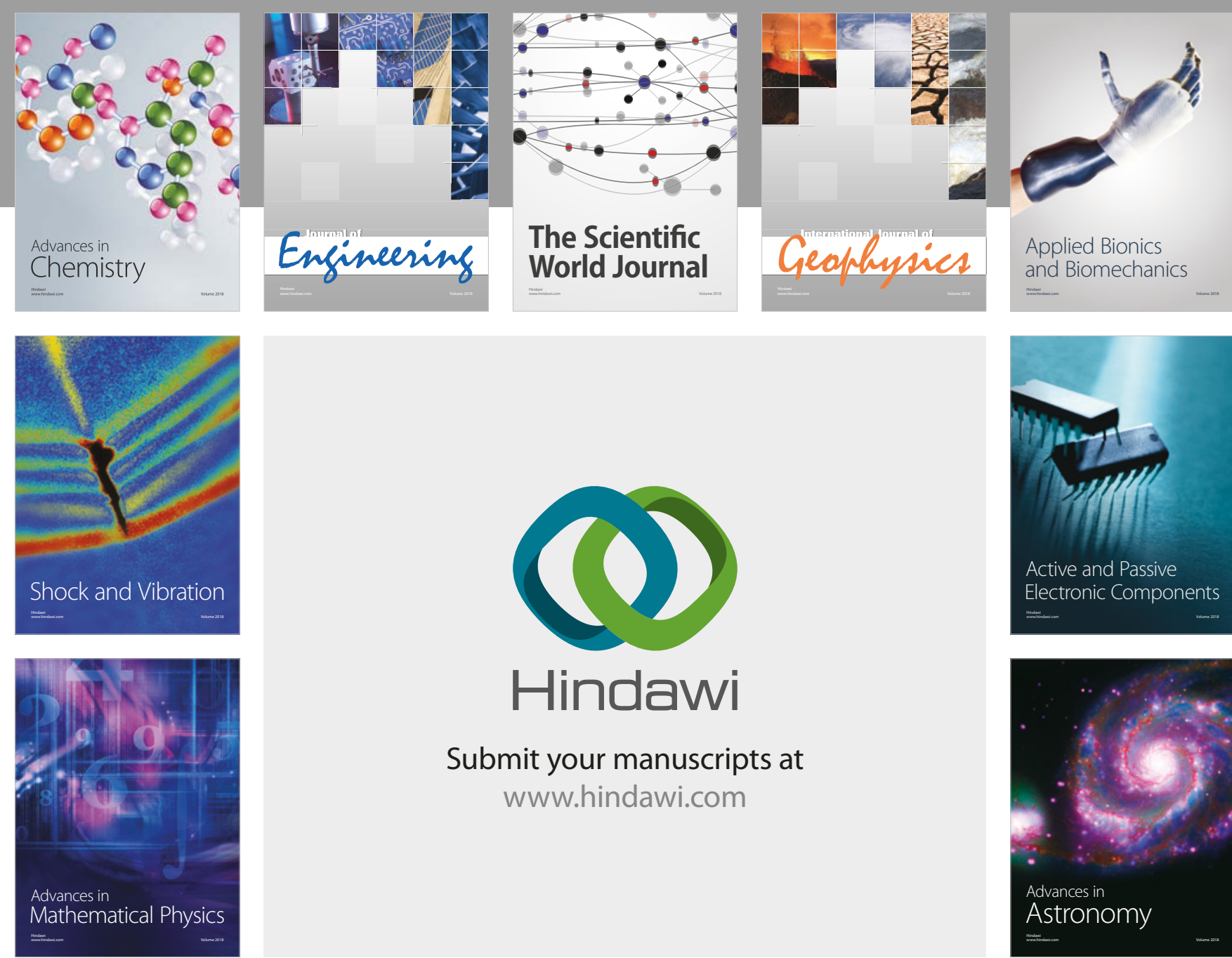

Submit your manuscripts at

www.hindawi.com

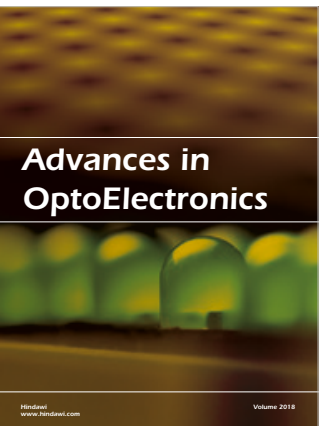

\section{Rotcting Machinery}
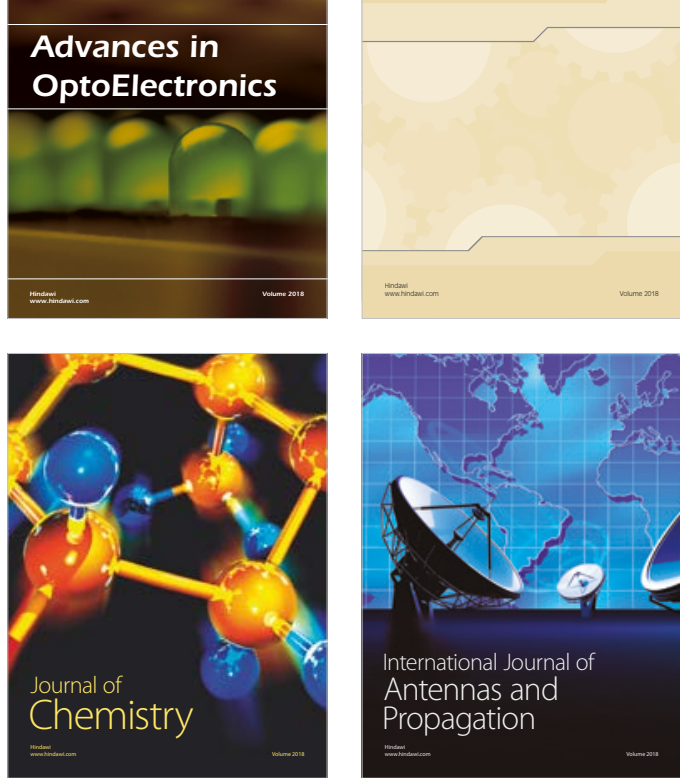

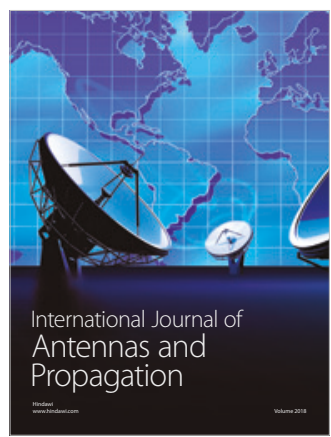

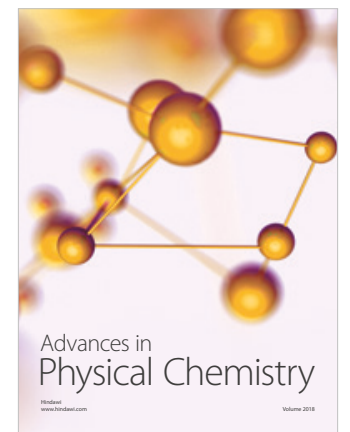

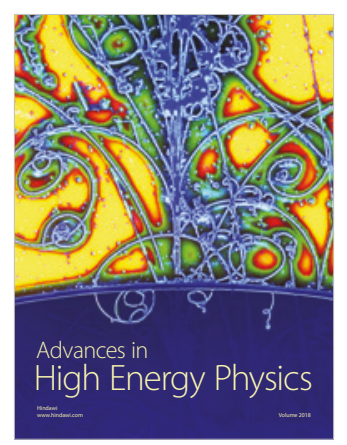

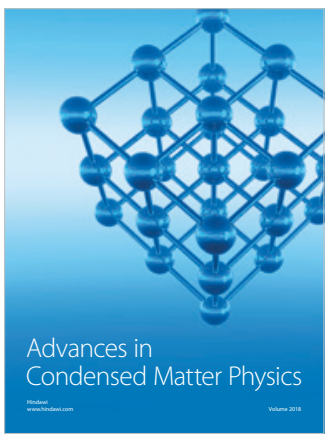

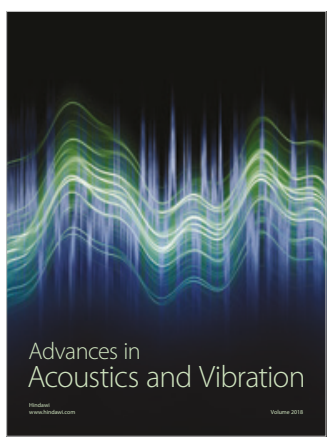

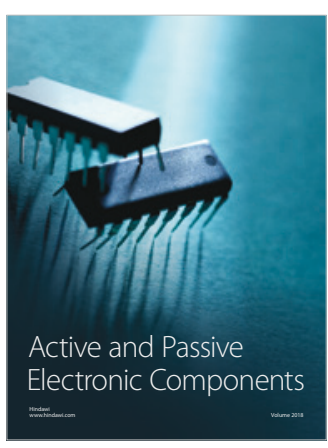
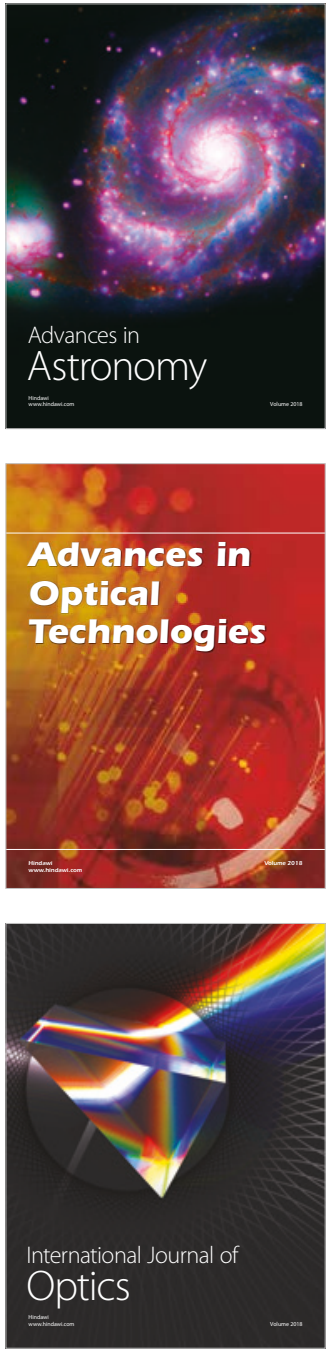